\title{
Effect of metformin versus placebo on metabolic factors in the MA.32 randomized breast cancer trial
}

Pamela J. Goodwin (iD ${ }^{1 凶}$, Ryan J. O. Dowling ${ }^{2}$, Marguerite Ennis $\mathbb{D}^{3}$, Bingshu E. Chen $\mathbb{I D}^{4}$, Wendy R. Parulekar ${ }^{4}$, Lois E. Shepherd ${ }^{4}$, Margot J. Burnell ${ }^{5}$, Rachel Vander Meer ${ }^{6}$, Andrea Molckovsky ${ }^{7}$, Anagha Gurjal ${ }^{8}$, Karen A. Gelmon $\mathbb{D}^{9}$, Jennifer A. Ligibel ${ }^{10}$, Dawn L. Hershman (D ${ }^{11}$, Ingrid A. Mayer ${ }^{12}$, Timothy J. Whelan ${ }^{13}$, Timothy J. Hobday ${ }^{14}$, Priya Rastogi ${ }^{15}$, Manuela Rabaglio-Poretti ${ }^{16}$, Julie Lemieux ${ }^{17}$, Alastair M. Thompson ${ }^{18}$, Daniel W. Rea ${ }^{19}$ and Vuk Stambolic ${ }^{20,21}$

Metformin may exert anticancer effects through indirect (mediated by metabolic changes) or direct mechanisms. The goal was to examine metformin impact on metabolic factors in non-diabetic subjects and determine whether this impact varies by baseline BMI, insulin, and rs11212617 SNP in CCTG MA.32, a double-blind placebo-controlled randomized adjuvant breast cancer (BC) trial. 3649 subjects with T1-3, N0-3, MO BC were randomized; pretreatment and 6-month on-treatment fasting plasma was centrally assayed for insulin, leptin, highly sensitive C-reactive protein (hsCRP). Glucose was measured locally and homeostasis model assessment (HOMA) calculated. Genomic DNA was analyzed for the rs11212617 SNP. Absolute and relative change of metabolic factors (metformin versus placebo) were compared using Wilcoxon rank and $t$-tests. Regression models were adjusted for baseline differences and assessed interactions with baseline BMI, insulin, and the SNP. Mean age was 52 years. The majority had T2/3, node positive, hormone receptor positive, HER2 negative BC treated with (neo)adjuvant chemotherapy and hormone therapy. Median baseline body mass index (BMI) was $27.4 \mathrm{~kg} / \mathrm{m}^{2}$ (metformin) and $27.3 \mathrm{~kg} / \mathrm{m}^{2}$ (placebo). Median weight change was $-1.4 \mathrm{~kg}$ (metformin) vs $+0.5 \mathrm{~kg}$ (placebo). Significant improvements were seen in all metabolic factors, with 6 month standardized ratios (metformin/placebo) of 0.85 (insulin), 0.83 (HOMA), 0.80 (leptin), and 0.84 (hsCRP), with no qualitative interactions with baseline BMI or insulin. Changes did not differ by rs11212617 allele. Metformin (vs placebo) led to significant improvements in weight and metabolic factors; these changes did not differ by rs11212617 allele status.

npj Breast Cancer (2021)7:74; https://doi.org/10.1038/s41523-021-00275-z

\section{INTRODUCTION}

The anti-diabetes drug metformin has been associated with reduced cancer risk and improved cancer prognosis in observational studies ${ }^{1,2}$; it may exert beneficial effects on cancer through both indirect and direct mechanisms. Indirect effects are likely mediated by systemic reductions in insulin, which results in decreased tumor-specific activation of the insulin receptor (IR) and suppression of mitogenic PI3K and Ras signaling ${ }^{3}$. Alternatively, metformin may exhibit direct inhibitory effects on cancer cells that are achieved by liver kinase B1 (LKB1)-mediated activation of AMPactivated protein kinase (AMPK), a negative regulator of PI3K/Akt/ mTOR signaling and protein synthesis ${ }^{4}$. While several clinical intervention studies have explored possible anticancer effects of metformin $^{5-9}$, none has assessed impact on patient outcome in the adjuvant setting. Canadian Cancer Trials Group (CCTG) MA.32 was initiated in 2010 to test the effect of metformin (versus placebo) on breast cancer (BC) outcomes in women with high-risk early-stage $\mathrm{BC}$ cancer who were receiving standard therapy.

We have previously reported ${ }^{10}$ improvements in body weight and circulating blood factors [glucose, insulin, leptin, highly sensitive
C-reactive protein (hsCRP), homeostasis model assessment (HOMA)] in a preplanned safety analysis of the first 492 MA.32 subjects. Here, we report an in-depth analysis of the effect of metformin versus placebo on change in body weight and circulating metabolic factors in the full MA.32 study population, exploring interactions with baseline BMI and insulin. In addition, we examine the impact of the rs11212617 single-nucleotide polymorphism (SNP) located near the ATM gene on these variables. Glycemic response to metformin has been linked to this SNP in some, but not all, studies ${ }^{11-14}$. Specifically, presence of the minor allele $(C)$ in diabetic patients has been associated with greater reduction in glycated hemoglobin (HbA1C). The status of this SNP has also been associated with metformin benefit in tumor response in human epidermal growth factor receptor 2 (HER2) positive $\mathrm{BC}^{15}$.

\section{RESULTS}

\section{Study population}

3649 women were randomized (1824 metformin, 1825 placebosee Fig. 1). Fasting blood was available at both baseline and on-

\footnotetext{
${ }^{1}$ Lunenfeld-Tanenbaum Research Institute, Mount Sinai Hospital, and Department of Medicine, University of Toronto, Toronto, ON, Canada. ${ }^{2}$ Hoffman-La Roche Limited, Mississauga, ON, Canada. ${ }^{3}$ Applied Statistician, Markham, ON, Canada. ${ }^{4}$ Canadian Cancer Trials Group, Queen's University, Kingston, ON, Canada. ${ }^{5}$ Department of Oncology, Saint John Regional Hospital, St. John, NB, Canada. ${ }^{6}$ Department of Oncology, Niagara Health System, St. Catharines, ON, Canada. ${ }^{7}$ Department of Medical Oncology, Grand River Regional Cancer Centre, Kitchener, ON, Canada. ${ }^{8}$ Abbotsford Centre, British Columbia Cancer Agency, Abbotsford, BC, Canada. ${ }^{9}$ University of British Columbia, BC Cancer Agency, Vancouver, BC, Canada. ${ }^{10}$ Dana-Farber Cancer Institute, Boston, MA, USA. ${ }^{11}$ Herbert Irving Comprehensive Cancer Center, Columbia University Medical Center, Columbia, NY, USA. ${ }^{12}$ Vanderbilt University, Nashville, TN, USA. ${ }^{13}$ McMaster University, Juravinski Cancer Centre, Hamilton, ON, Canada. ${ }^{14}$ Mayo Clinic, Rochester, MN, USA. ${ }^{15}$ NRG Oncology and University of Pittsburgh Medical Center, Pittsburgh, PA, USA. ${ }^{16}$ IBCSG and Department of Oncology, Bern University Hospital, University of Bern, Berne, Switzerland. ${ }^{17} \mathrm{CHU}$ de Québec-Université Laval, Québec, QC, Canada. ${ }^{18}$ Baylor College of Medicine, Houston, TX, USA. ${ }^{19}$ Cancer Research UK Clinical Trials Unit (CRCTU), Institute of Cancer and Genomic Sciences, University of Birmingham, Birmingham, UK. ${ }^{20}$ Princess Margaret Cancer Centre, University Health Network, Toronto, ON, Canada. ${ }^{21}$ Department of Medical Biophysics, University of Toronto, Toronto, ON, Canada. ${ }^{凶}$ email: Pamela.Goodwin@sinaihealthsystem.ca
} 


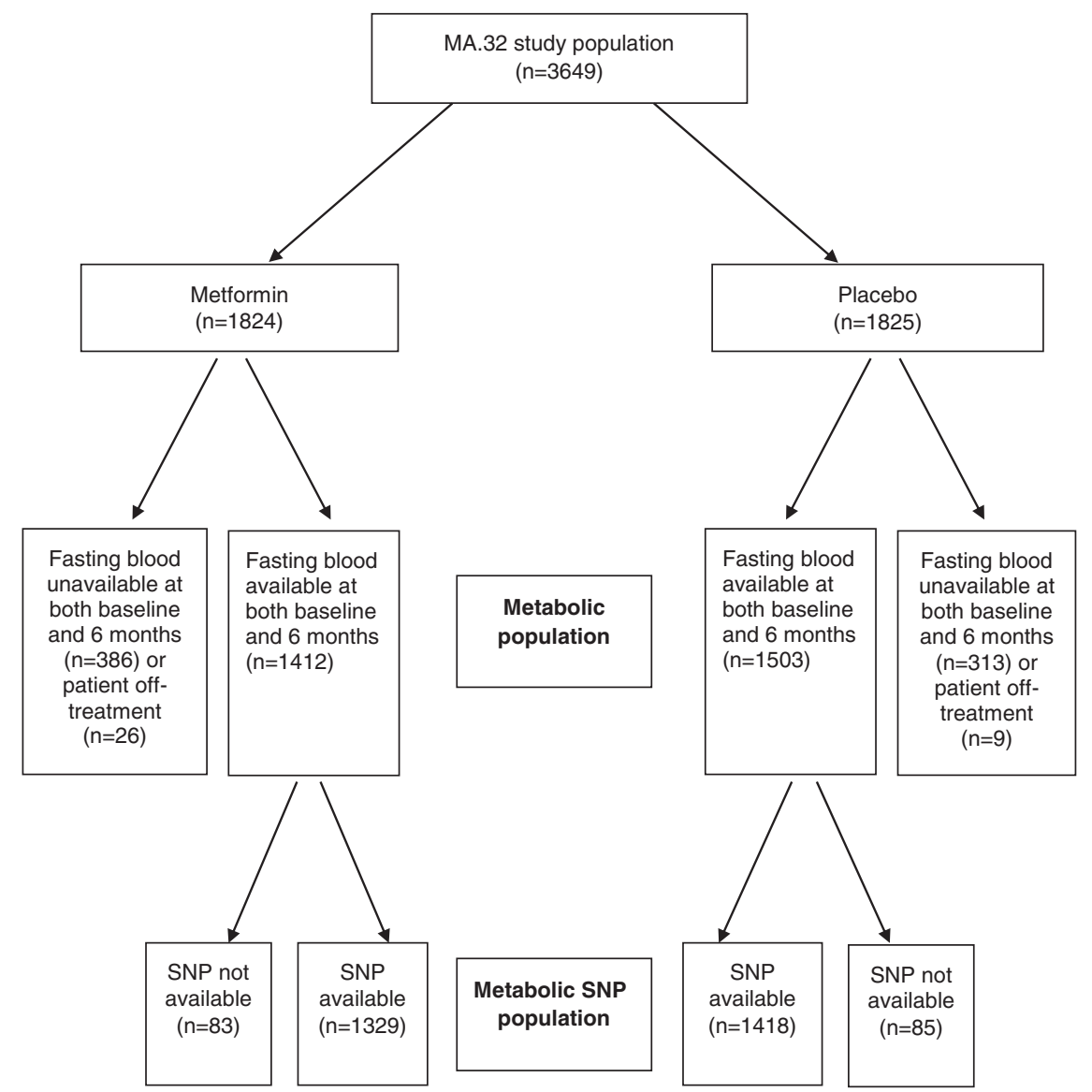

Fig. 1 CONSORT diagram-identification of subjects included in the metabolic and metabolic SNP populations from the full MA.32 study population. SNP single-nucleotide polymorphism.

treatment at 6 months in 1412 metformin and 1503 placebo subjects; these subjects represent the metabolic population in this paper. SNP information was available on 1329 of the metformin patients and 1418 of the placebo patients-these patients represent the metabolic SNP population.

Baseline characteristics of the subjects included in, and excluded from, the metabolic analyses are shown in Table 1. It can be seen that subjects included (vs excluded) in the metabolic analyses were less likely to have been randomized to metformin (48.4\% included vs $56.1 \%$ excluded) than placebo (51.6 vs 43.9 , $p<0.001$ compared to metformin), less likely to be African American $(3.8 \%$ vs $7.5 \%, p<0.001)$, more likely to have had hormone receptor-positive breast cancer $(70.5 \%$ vs $65 \%, p=$ $0.004)$ and to have received hormone therapy $(62.7 \%$ vs $56.1 \%$, $p=0.003)$. They were less likely to have received neoadjuvant chemotherapy $(19.7 \%$ vs $26.3 \%, p<0.001)$; receipt of any adjuvant chemotherapy was similar in included and excluded subjects ( $89.3 \%$ vs $88.8 \%$ ). The rs 11212617 SNP genotyping was performed on $94.2 \%$ of the patients in the metabolic population.

Mean age of included subjects was 52 years, the majority (91\%) were white with clinical or pathologic T2 or T3 tumors $(67.4 \%$ metformin, $65.3 \%$ placebo) that were node-positive $(56.4 \%$ metformin, $54.4 \%$ placebo), hormone receptor-positive (70.6\% metformin, $70.5 \%$ placebo), and HER2 negative $(82.8 \%$ metformin, $83 \%$ placebo). The majority received adjuvant chemotherapy (89.9\% metformin, $88.8 \%$ placebo) and hormone therapy $(62.7 \%$ in both arms). Adjuvant trastuzumab use was similar in both arms (17.4\% metformin, $17.2 \%$ placebo). Just over one-third $(34.3 \%$ metformin, $33.8 \%$ placebo) had $\mathrm{BMI} \geq 30 \mathrm{~kg} / \mathrm{m}^{2}$ at baseline.

The AA genotype of the rs11212617 SNP was present in $30 \%$ of the metabolic population, $A C$ in $49 \%$ and CC in $21 \%$; by-arm distributions were similar. A and $C$ allelic frequencies were 0.54 and 0.46 , respectively, in accordance with Hardy Weinberg equilibrium $\left(X^{2}=0.35, p=0.55\right)$. When patients were classified by self-reported race/ethnicity, the genotype frequencies differed significantly among groups $(p \leq 0.001)$, A being present in the majority of Whites (80.1\%) and $C$ in the majority of African Americans (86.3\%). Specific frequencies for $A A, A C$, and CC were $30.7 \%, 49.4 \%$, and $19.9 \%$ in 2530 White, $25.4 \%, 50.7 \%$, and $23.9 \%$ in 67 Asian, $13.7 \%, 36.8 \%$, and $49.5 \%$ in 95 African American, $20.8 \%, 62.5 \%$, and $16.7 \%$ in 24 American Indian, Alaskan Native, Native Hawaiian or other Pacific Islander subjects, and $38.7 \%$, $41.9 \%$, and $19.4 \%$ in 31 subjects with non-reported ethnicity, respectively.

\section{Baseline and 6-month change in metabolic factors}

Baseline values, raw differences and relative change from baseline to 6 months are shown in Table 2 for body size and circulating metabolic factors. Month 6 standardized metformin to placebo ratios, adjusted for baseline differences in the variable, BMI and age are also shown. Baseline values of all variables were similar in metformin vs placebo arms. At 6 months, there were significant raw and relative improvements in all variables in metformin versus placebo subjects. Metformin subjects lost a median of $1.4 \mathrm{~kg}$ (vs $0.5 \mathrm{~kg}$ gain in placebo subjects, $p<0.0001$; relative change $-2 \%$ metformin vs $+1 \%$ placebo, $p<0.0001)$. In univariable analyses, metformin (vs placebo) subjects experienced reductions in glucose (raw change $-0.1 \mathrm{vs}+0.1 \mathrm{mmol} / \mathrm{L}, p<0.0001$; relative change $-2 \%$ vs $+1 \%, p<0.0001$ ), insulin (raw change -7 vs $+2 \mathrm{pmol} / \mathrm{L}, p<$ 0.0001 ; relative change $-11 \%$ vs $+6 \%, p<0.0001$ ), HOMA (raw change -0.2 vs $+0.1, p<0.0001$; relative change $-10 \%$ vs $+11 \%$, 
Table 1. Baseline patient and tumor characteristics.

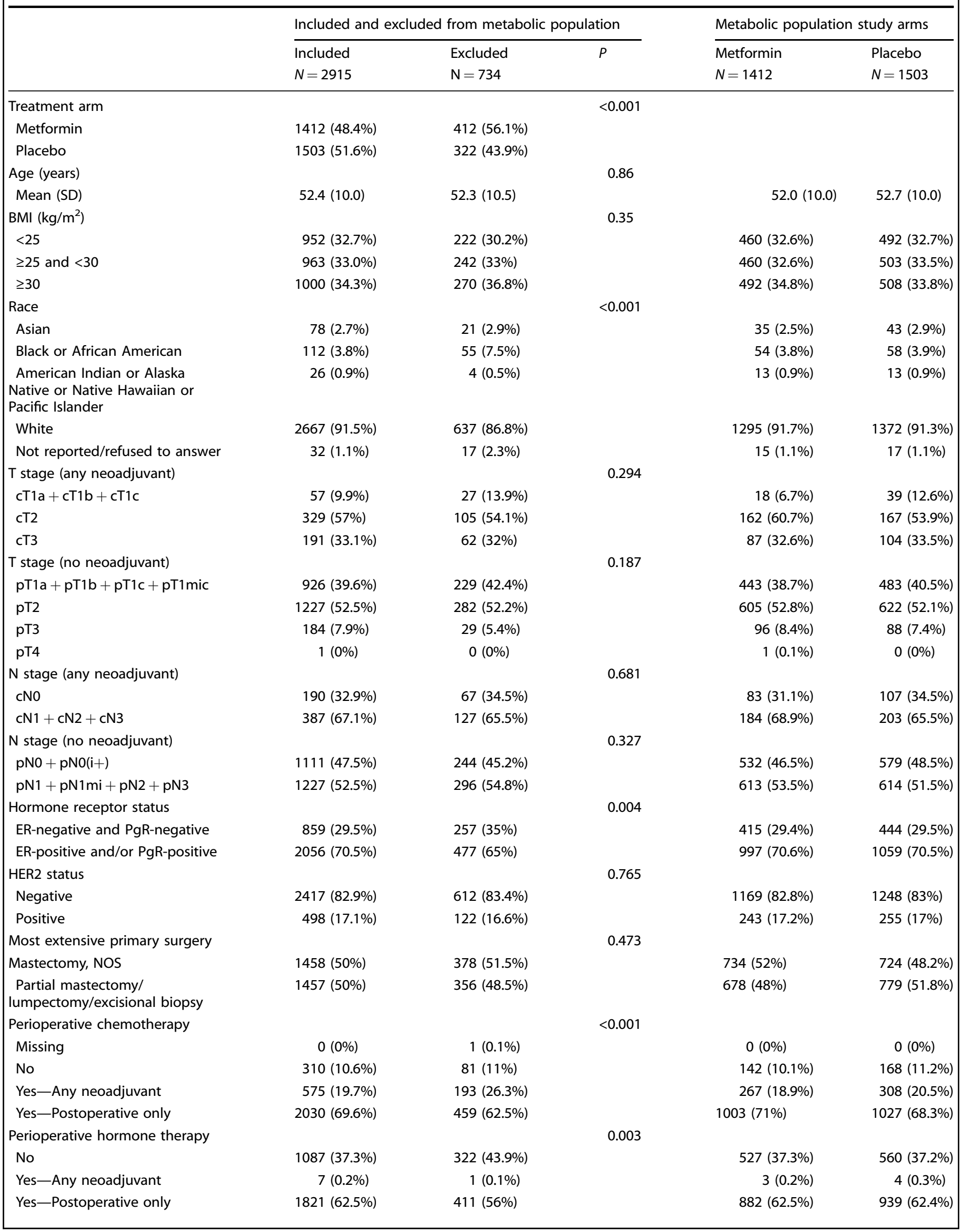


Table 1 continued

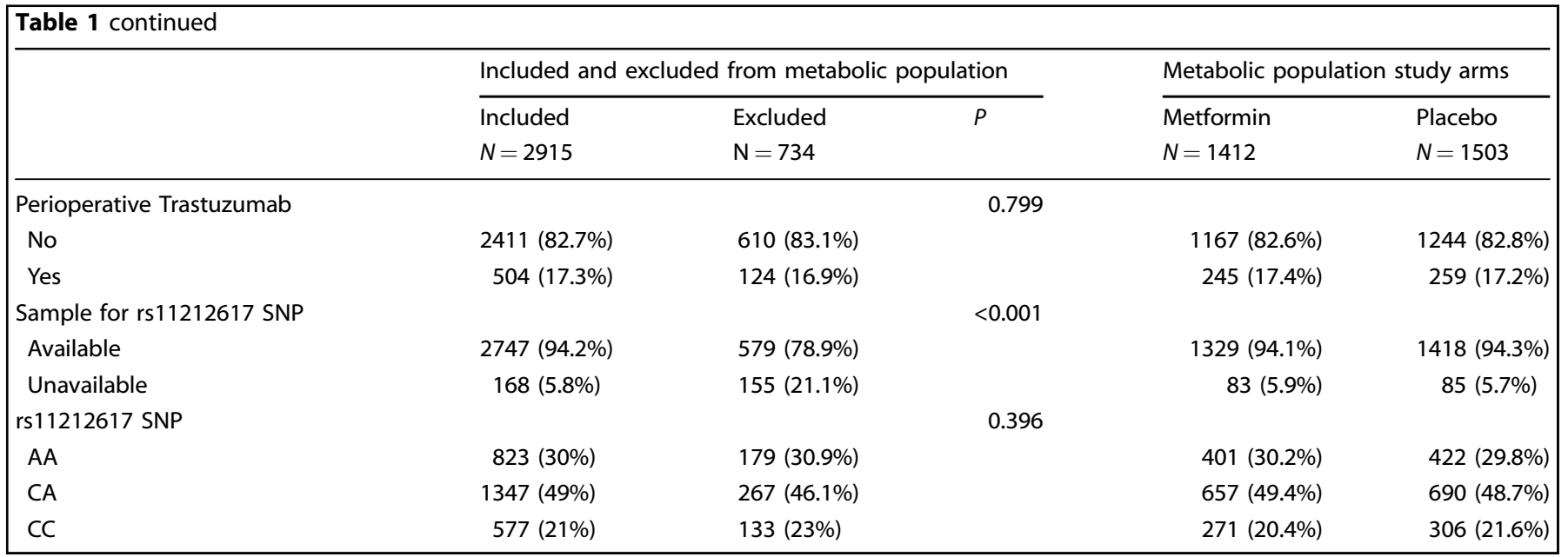

$p<0.0001$ ), leptin (raw change -0.8 vs $+1.1 \mathrm{ng} / \mathrm{ml}, p<0.0001$; relative change $-10 \%$ vs $+12 \%, p<0.0001$ ), and hsCRP (raw change -0.1 vs $+0.1 \mu \mathrm{g} / \mathrm{L}, p<0.0001$; relative change $-9 \%$ vs $+10 \%, p<0.0001)$. After adjustment for baseline variable levels, $\mathrm{BMI}$ and age, the standardized 6-month metformin to placebo ratios were 0.97 for weight or $\mathrm{BMI}, 0.98$ for glucose, 0.85 for insulin, 0.83 for HOMA, 0.80 for leptin, and 0.84 for hsCRP (all $p<0.0001$ compared to a ratio of 1 ). This reflects, for example, 6-month insulin levels being 15\% lower in the metformin arm than in the placebo arm when standardized to patients with the same baseline insulin, age, and BMI.

There was little evidence of a significant interaction of change in these variables with baseline BMI or insulin. The standardized metformin to placebo ratios at 6 months for each variable according to baseline BMI and baseline insulin are shown in Fig. 2 panels $a$ and $b$. As can be seen, potential quantitative interactions were identified for baseline BMI with leptin (interaction $p=0.02$, standardized metformin to placebo ratio 0.75 when $\mathrm{BMI}=20 \mathrm{~kg} /$ $\mathrm{m}^{2}$ and 0.81 for $\mathrm{BMI}=30 \mathrm{~kg} / \mathrm{m}^{2}$ ) and for baseline insulin with hsCRP (interaction $p=0.04$, standardized ratio 0.79 for insulin $=$ $40 \mathrm{pmol} / \mathrm{L}$ and 0.88 for insulin $=100 \mathrm{pmol} / \mathrm{L}$ ). Similarly, there was no evidence that change in body size or blood variables differed in those receiving adjuvant endocrine therapy vs no (all interaction $P \geq 0.22$, see Supplemental Table 1).

\section{rs11212617 SNP}

At baseline, a pattern of increasing weight, BMI, and leptin was seen according to the number of rs $11212617 \mathrm{C}$ alleles (Table 3): median weights for the three groups $A A, A C$, and $C C$ are 72.0, 73.3, and $75.4 \mathrm{~kg}(p=0.01)$; median BMI 27.0, 27.4 , and $28.2 \mathrm{~kg} / \mathrm{m}^{2}(p=$ $0.02)$; median leptin 12.0, 12.6, and $13.4 \mathrm{ng} / \mathrm{ml}(p=0.05)$.

Examination of interactions between the study drug (metformin versus placebo) and the SNP status (any $C$ versus $A A$ ) showed no evidence that metformin was more effective on body size or metabolic factors when the $C$ allele was present (Fig. 2c, all interaction $P>0.21$ ).

\section{DISCUSSION}

Metformin (versus placebo) was associated with improvement in body size as well as a range of circulating metabolic markers in the non-diabetic population that was enrolled onto MA.32. For the most part, these improvements with metformin were independent of baseline BMI and insulin levels and did not vary by rs11212617 genotype. Although the borderline significant interactions of baseline BMI with change in leptin and of insulin with change in hsCRP may have been due to chance (multiple interactions were tested for multiple variables) they warrant evaluation in future studies; it is important to note that these potential interactions were quantitative, not qualitative with metformin (vs placebo) benefits being consistently observed. These findings suggest that the improvement in metabolic factors seen with metformin is broad-based. Furthermore, the relative changes in metformin vs placebo subjects that we identified (e.g., a 15\% lower insulin in metformin relative to placebo subjects at 6 months) were clinically significant and consistent with our hypothesized effects. Together, our findings support the study of metformin in both metabolically healthy and unhealthy $\mathrm{BC}$ patients and they are consistent with potential beneficial indirect effects of metformin on BC outcomes. In our upcoming efficacy analysis of MA.32 we will analyze the extent to which metabolic response to metformin is associated with BC outcomes. Our observations also suggest metformin may exert beneficial effects on cardiovascular and metabolic outcomes; this will be examined in our upcoming analysis.

Our results are consistent with two prior reports, in which metformin $500 \mathrm{mg}$ po tid was given for 6 months to $B C$ survivors ${ }^{17}$ or for 2-3 weeks in a BC neoadjuvant window of opportunity study $^{6}$, with change measurements taken while subjects were still taking metformin. In both studies, significant improvements were identified in weight, BMI and the metabolic factors reported here. In the neoadjuvant study, reductions in intra-tumoral ki67 that were independent of baseline BMI and HOMA were also seen. Similar improvements in metabolic status were reported by Kalinsky et al. ${ }^{18}$ in another neoadjuvant window of opportunity study in which subjects received metformin $500 \mathrm{mg}$ in the morning and $1000 \mathrm{mg}$ in the evening for a median of 23 days, although no associated reductions were seen in intra-tumoral ki67. In contrast, in a third BC neoadjuvant window of opportunity study ${ }^{19}$, there were no improvements in metabolic variables in patients receiving metformin $1700 \mathrm{mg}$ once daily after dinner for 4 weeks when metformin was discontinued $36-48 \mathrm{~h}$ before final measurements. Given a metformin half-life of $\sim 6 \mathrm{~h}$, the once-daily dosing schedule, coupled with the longer interval between drug discontinuation and final measurements may have obscured metabolic effects. Regardless, significant reductions in intratumoral ki67 were seen in individuals receiving metformin who had baseline insulin resistance (as defined by HOMA).

Our failure to identify an association of metabolic improvement with the rs11212617 SNP genotype was somewhat unexpected since some prior reports ${ }^{11,12,14}$ have identified the $C$ allele to be associated with enhanced glycemic response to metformin and/or higher plasma levels of the drug in diabetic patients. It is possible the differential glycemic effect of the $C$ vs $A$ allele is present only in diabetic subjects (MA.32 subjects were non-diabetic). The $C$ allele has been associated with enhanced pathologic complete 


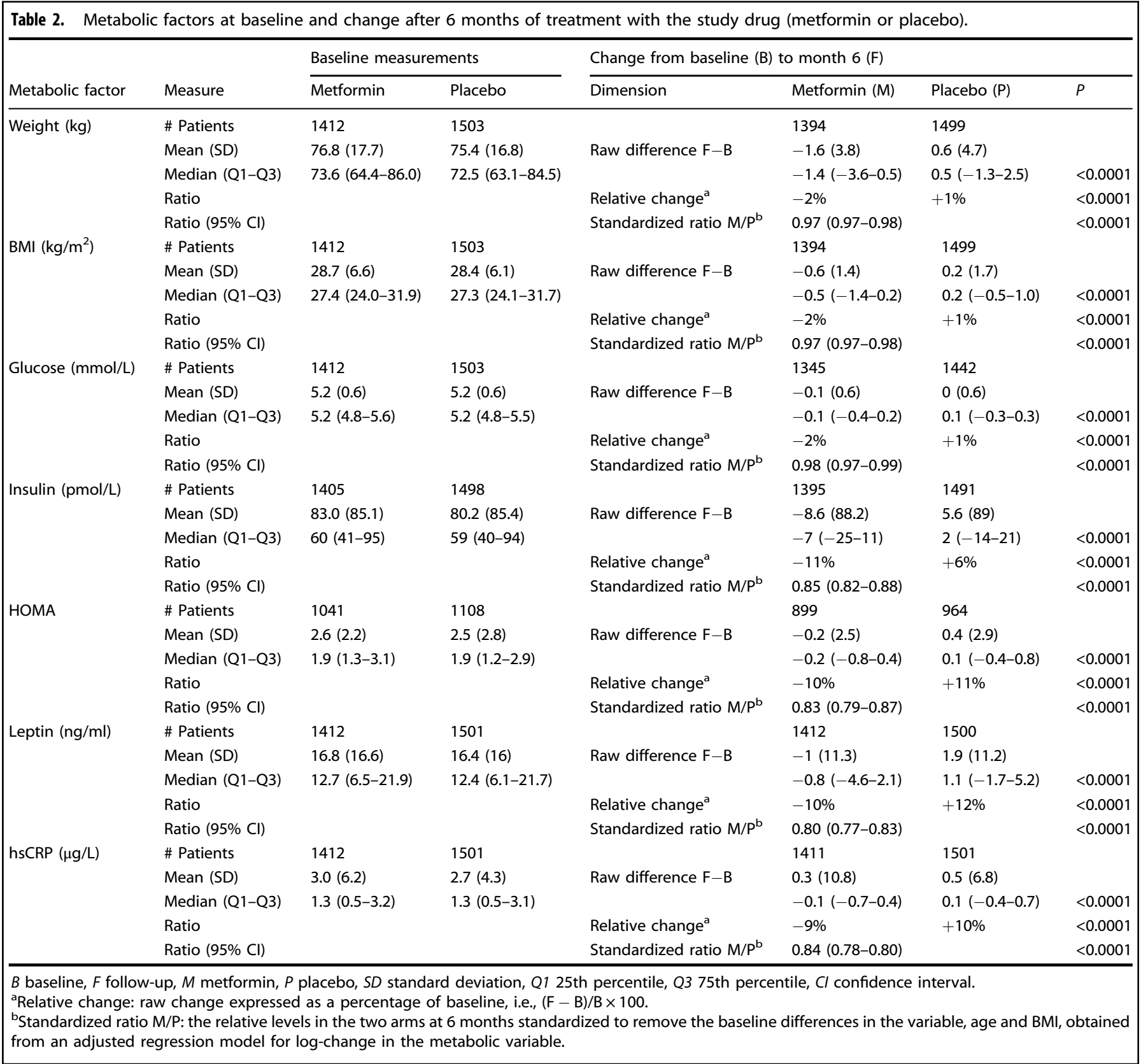

response to neoadjuvant chemotherapy/HER2 targeted treatment in non-diabetic HER2 $+B C$ subjects ${ }^{15}$ receiving metformin; this SNP associated effect may have been mediated by mechanisms that differed from the metabolic mechanisms we investigated here. Our observation that the $C$ allele is the major allele in African Americans (versus A in White/Caucasian populations) is novel. As a result, it is possible metformin effects or metformin toxicity will differ by race/ethnicity. Indeed, increased treatment success (reductions in $\mathrm{HbA1C}$ ) has been reported in African Americans with type 2 diabetes receiving metformin ${ }^{20}$; African Americans in the Diabetes Prevention Program randomized to metformin exhibited greater reductions (though non-significant) in diabetes incidence when compared to White subjects ${ }^{21}$. We plan to examine whether rs11212617 status and race are predictors of metformin effect on BC outcomes in MA.32 and we will examine metformin toxicity in relation to SNP status.

Strengths of our study include the prospective randomized design, large sample size, and standardization of blood handling and analysis. Limitations include the lack of data on more sensitive measures of insulin sensitivity, such as the frequently sampled intravenous glucose tolerance test. Furthermore, although subjects were required to be receiving study drug at the time of the 6-month measurement, some subjects may not have fully complied with drug dosing in the days leading up to the 6-month blood draw. We were not able to explore the potential contribution of metformin-associated toxicity to the changes in body size and metabolic factors we have studied; data on toxicity and compliance will be available after the planned efficacy analysis and these issues will be explored at that time. In addition, because information on diet and physical activity was not available on the majority of subjects ( $>75 \%)$, we were not able to explore the impact of lifestyle on change in body size and metabolic factors.

In conclusion, the administration of metformin $850 \mathrm{mg}$ po bid to non-diabetic early-stage breast cancer patients led to weight loss and improved metabolic health. These improvements were 
(a)

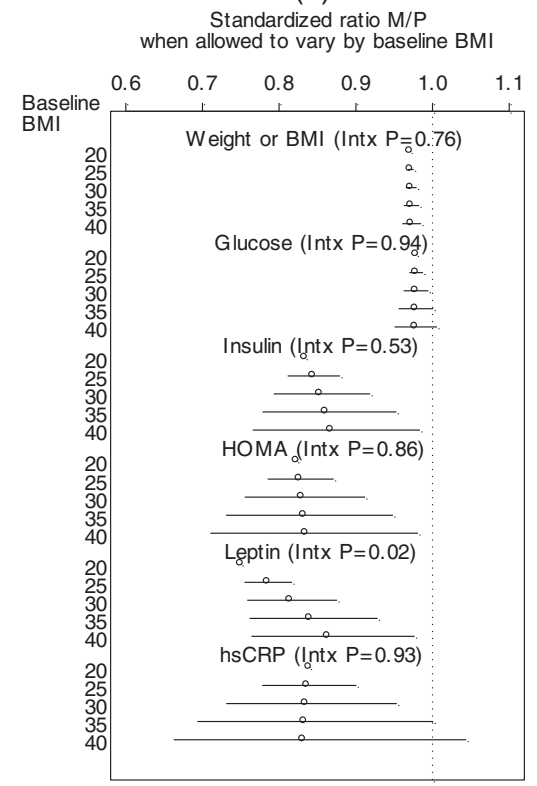

(b)

Standardized ratio $\mathrm{M} / \mathrm{P}$ when allowed to vary by baseline insulin

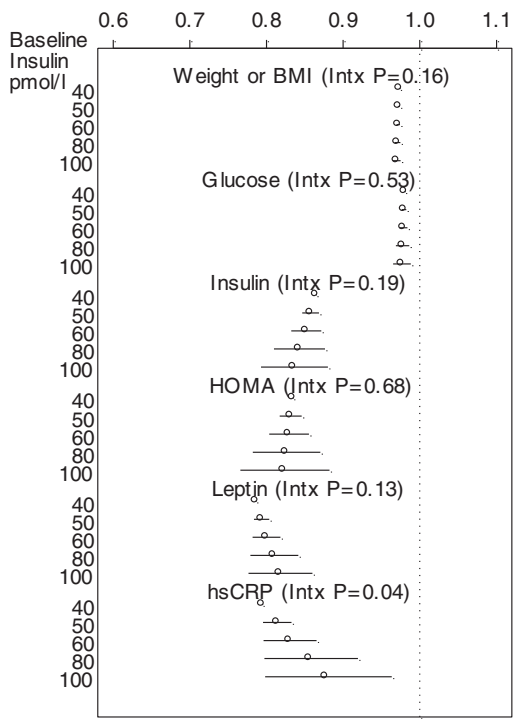

(c)

Standardized ratio $\mathrm{M} / \mathrm{P}$ en allowed to vary by SNP genotype

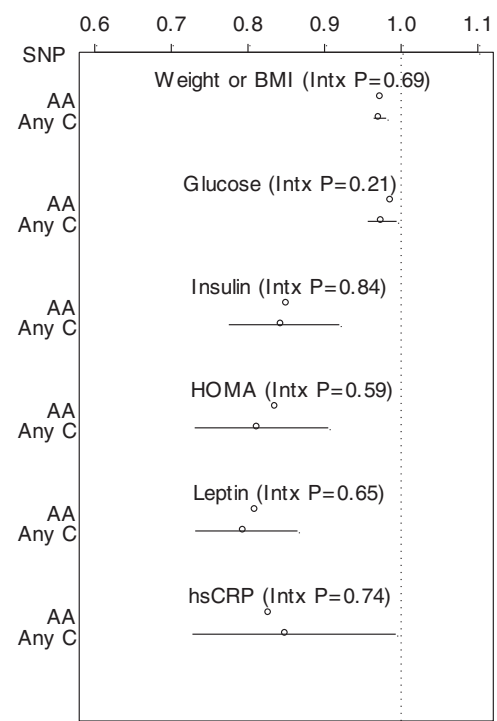

Fig. 2 Assessment of differential study drug effects on the metabolic outcomes weight/BMI, glucose, insulin, HOMA, leptin, and hsCRP, by baseline BMI, insulin, and the rs11212617 SNP. Depicted is the standardized metformin to placebo ratio of the metabolic factor at 6 months, with 95\% confidence intervals, obtained from adjusted interaction regression models. BMI body mass index, SNP single-nucleotide polymorphism, Intx interaction. a Interaction of study drug by baseline BMI (continuous). b Interaction of study drug by baseline insulin (continuous). c Interaction of study drug by genotype any C versus AA of the rs11212617 SNP.

largely independent of baseline BMI and insulin and did not differ by rs11212617 SNP status. They provide support for a potential indirect of metformin on breast cancer outcomes (mediated by reductions in insulin and other metabolic markers). Their potential contribution to metformin effects on breast cancer outcomes, as well as on cardiovascular disease and diabetes, will be examined in upcoming efficacy analyses, strengthening the clinical utility of our results.

\section{METHODS}

\section{Study design}

The CCTG MA.32 Clinical Trial (Clinical Trials.gov identifier: NCT01101438; http://clinicaltrials.gov/show/NCT01101438, first posted April 12, 2010) is a Phase III, randomized trial that enrolled 3649 non-diabetic subjects between 2010 and 2013; subjects received standard surgical, chemotherapeutic (completed at least one month prior to enrollment), hormonal, biologic, and radiation therapy for a T1-3, N0-3, MO BC diagnosed during the previous year. Subjects with T1C NO BC were eligible if they had at least one of histologic grade III, lymphovascular invasion, negative estrogen (ER) and progesterone $(\mathrm{PgR})$ receptors, HER2 positivity, Oncotype Recurrence Score $\geq 25$ or Ki-67 over 14\%. In May 2012, after 2382 subjects were enrolled, eligibility criteria were amended to mandate triple negative (ERnegative, PgR-negative, HER2 negative) status for patients with T1cNO disease and at least one of the above adverse tumor characteristic for patients with T2NO tumors. Participants were required to have a fasting glucose of $7.0 \mathrm{mmol} / \mathrm{L}$ or lower; those with a history of diabetes, lactic acidosis, current use of diabetes medication, breast cancer recurrence or previous invasive cancer, excessive alcohol intake, or marked hepatic, kidney, or cardiac dysfunction were excluded.

Eligible subjects were randomly assigned (1:1, using computergenerated randomization) in a double-blind fashion to metformin $850 \mathrm{mg}$ caplet po bid or an identical placebo po bid for 5 years, including a 4-week ramp-up of one caplet per day. Height and weight were measured (in indoor clothing, without shoes) at study centers at baseline and weight at 6 months. The primary study outcome, invasive disease-free survival, as well as secondary outcomes, including overall survival and breast cancer-free interval, have not yet been reported.
The study protocol was approved by institutional review boards of participating institutions, including the $\mathrm{NCl}$ (US) Central Institutional Review Board and Mount Sinai Hospital (Ontario Cancer Research Ethics Board). All patients provided written informed consent to participate.

\section{Laboratory analyses}

At baseline (before starting study therapy) blood was drawn into a lithium heparin tube after an overnight fast of at least $12 \mathrm{~h}$ and centrifuged at $1500 \mathrm{rpm}$ for $10 \mathrm{~min}$; plasma was aliquoted and frozen within $30 \mathrm{~min}$ of collection at $-80^{\circ} \mathrm{C}$ at the local center. Aliquots were transported on dry ice to the central repository at CCTG in Kingston, Canada. Specimens were re-transported on dry ice to Mount Sinai Hospital in Toronto for analysis. Paired bloods (baseline, 6 months) were assayed (blinded to treatment allocation, without dilution) in batches with $10 \%$ random repeats for insulin (Roche ElectroChemiLuminescence Immunoassay (ECLIA), leptin (Luminex Milliplex MAP assay), and hsCRP (Roche, particle-based immunoturbidimetric assay). Blood was analyzed in 2014-2015. Intraassay coefficients of variability were $3 \%, 3 \%$, and $4 \%$ for insulin, leptin, and hsCRP, respectively. Glucose was analyzed at local clinical laboratories immediately after collection. HOMA (a marker of insulin resistance) was calculated from glucose and insulin levels when both were measured on the same day [glucose $(\mathrm{mg} / \mathrm{dl}) \times$ insulin $(\mathrm{pmol} / \mathrm{L}) / 22.5]^{16}$.

Blood for genomic analysis was drawn into EDTA tubes that were aliquoted into $1.5 \mathrm{ml}$ cryovials and stored as described above. One aliquot was sent on dry ice for genomic DNA extraction and genotyping for the SNP rs11212617 (Chr11(GRCh38):g.108412434C>A) at The Centre for Applied Genomics, Hospital for Sick Children, Toronto, Canada, using a QIAsymphony magnetic bead DNA extractor (Qiagen, Germany) and PCR primers (5'ACAAACAGGAAACAATTACAAATACAATAAAT3' and 5'TTAAAGT GGGTTGCTTGTGGATAA3') with TaqMan ${ }^{\circledR} 100 \mathrm{mM}$ dual-label MGB probes AGATCAGAGACTGTCAGAGC and AGATCAGAGAATGTCAGAGC (Applied Biosystems $\mathrm{s}^{\mathrm{TM}}$, ThermoFisher Scientific, Waltham, $\bar{M} A$, USA). The reaction mix consisted of $5 \mu \mathrm{l} \mathrm{TaqMan}{ }^{\circledR}$ Genotyping Master Mix (Life Technologies), $1 \mu \mathrm{l}$ of each PCR primer (each at $10 \mathrm{mM}$ ), $0.02 \mu \mathrm{l}$ of each probe, $2.0 \mu \mathrm{l}$ water, and 20-50 ng of DNA template. Samples were analyzed using the $\mathrm{ViiA}^{\mathrm{TM}} 7$ Real-Time PCR System (Applied Biosystems ${ }^{T M}$ ) and analyzed using ViiA ${ }^{T M}$ 7 software. Each genotyping run contained 92 study samples along with Coriell reference samples NA12878 (C/A), NA12813 (A/A), NA19240 (C/C), and a no template control. 
Table 3. Metabolic factors at baseline broken down by the rs 11212617 SNP.

\begin{tabular}{|c|c|c|c|c|c|c|c|c|}
\hline Metabolic factor & Measure & AA & $A C$ & CC & $P^{a}$ & AA & Any $C$ & $P^{a}$ \\
\hline$(\mathrm{kg})$ & Mean (SD) & $75.4(17.1)$ & $76.1(16.8)$ & $78.1(18.7)$ & & $75.4(17.1)$ & 76.7 (17.4) & \\
\hline BMI & \# Patients & 823 & 1347 & 577 & & 823 & 1924 & \\
\hline$\left(\mathrm{kg} / \mathrm{m}^{2}\right)$ & Mean (SD) & $28.3(6.3)$ & $28.5(6.1)$ & $29.3(7)$ & & $28.3(6.3)$ & $28.8(6.4)$ & \\
\hline \multirow[t]{2}{*}{$(\mathrm{mmol} / \mathrm{L})$} & Mean (SD) & $5.2(0.6)$ & $5.2(0.5)$ & $5.2(0.6)$ & & $5.2(0.6)$ & $5.2(0.6)$ & \\
\hline & Median (Q1-Q3) & $5.2(4.8-5.6)$ & $5.2(4.8-5.5)$ & $5.2(4.8-5.6)$ & 0.40 & $5.2(4.8-5.6)$ & $5.2(4.8-5.5)$ & 0.22 \\
\hline Insulin & \# Patients & 820 & 1344 & 573 & & 820 & 1917 & \\
\hline \multirow[t]{2}{*}{ (pmol/L) } & Mean (SD) & $76.3(69.1)$ & $83.0(91.2)$ & $86.2(93.3)$ & & $76.3(69.1)$ & 83.9 (91.8) & \\
\hline & Median (Q1-Q3) & 59 (40-94) & 60 (41-94) & $60(40-97)$ & 0.76 & $59(40-94)$ & $60(40-94)$ & 0.47 \\
\hline \multirow[t]{2}{*}{$(\mathrm{ng} / \mathrm{ml})$} & Mean (SD) & $15.9(16.5)$ & $16.2(14.5)$ & $18.7(19.6)$ & & $15.9(16.5)$ & $17(16.2)$ & \\
\hline & Median (Q1-Q3) & $12.0(6-21.3)$ & $12.6(6.4-21.2)$ & $13.4(6.2-24.4)$ & 0.05 & $12.0(6-21.3)$ & $12.7(6.3-22.1)$ & 0.07 \\
\hline hsCRP & \# Patients & 823 & 1346 & 576 & & 823 & 1922 & \\
\hline \multirow[t]{2}{*}{$(\mu \mathrm{g} / \mathrm{L})$} & Mean (SD) & $3.0(7.2)$ & $2.9(4.7)$ & $2.8(3.9)$ & & $3.0(7.2)$ & $2.9(4.5)$ & \\
\hline & Median (Q1-Q3) & $1.2(0.5-3)$ & $1.3(0.5-3.3)$ & $1.5(0.6-3.3)$ & 0.25 & $1.2(0.5-3)$ & $1.4(0.5-3.3)$ & 0.28 \\
\hline
\end{tabular}

\section{Statistical analyses}

Statistical analyses were conducted by Drs. Bingshu Chen (CCTG) and Marguerite Ennis using SAS version 9.2. Patient and tumor characteristics at baseline were tabulated by study arm and compared using $x^{2}$ tests for categorical variables and Wilcoxon rank-sum tests for continuous variables. These tests were performed because selection criteria for this study included post-randomization criteria.

Change from baseline (B) to follow-up (F) after 6 months of treatment with metformin or placebo was assessed. Summary statistics for raw change (follow-up minus baseline, $\mathrm{F}-\mathrm{B}$ ) were tabulated by arm and compared using Wilcoxon rank-sum tests. All the metabolic factors had skew distributions which were greatly improved by applying log transformations. The average log-change (average of $\log (\mathrm{F})-\log (\mathrm{B})$ ) was calculated and the arms compared via $t$-tests. An effect size measure was obtained by back-transforming the logchange averages to geometric means $\mathrm{F} / \mathrm{B}$ and calculating percent relative change as $(F-B) / B \times 100$. Thus the relative change denotes raw within-arm change. Further comparisons that adjusted for baseline age, BMI, and baseline levels of the metabolic variable were performed using linear regression models with log-change as an outcome. Results are presented as standardized ratios by back-transforming the study arm parameter. Because of the baseline adjustment, these ratios give the relative levels in the two arms at 6 months "standardized" to remove any baseline differences between the two arms in the variable, age and BMI. Note that when using ratios the same results are obtained for $\mathrm{BMl}$ as for weight because height cancels out in the BMI ratios.

The above regression models were expanded with suitable interaction terms to explore whether the study drug had a differential effect on a metabolic outcome depending on (1) baseline BMI level, (2) baseline insulin level, and (3) the genotype any $C$ versus AA of the rs11212617 SNP.

Measures of effect size and uncertainty were provided. A $p$ value $\leq 0.05$ was considered statistically significant and all tests were two-sided.

\section{Reporting summary}

Further information on research design is available in the Nature Research Reporting Summary linked to this article.

\section{DATA AVAILABILITY}

The data generated and analyzed during this study are described in the following data record: https://doi.org/10.6084/m9.figshare.14447598 ${ }^{22}$. The SNP data are openly available as part of the data record. The primary efficacy analysis will be available from the Canadian Cancer Trials Group (Kingston, Ontario) after the results of the analysis have been published. These data will be uploaded to the $\mathrm{NCl}$ data archive website: https://nctn-data-archive.nci.nih.gov/view-trials and will be searchable via NCT trial number NCT01101438. As of April 2021, the group is working towards this publication. Further details can be requested from the corresponding author. The clinical data are not publicly available for the following reason: data contain information that could compromise research participant privacy.

Received: 21 December 2020; Accepted: 3 May 2021; Published online: 08 June 2021

\section{REFERENCES}

1. Libby, G. et al. New users of metformin are at low risk of incident cancer: a cohort study among people with type 2 diabetes. Diabetes Care 32, 1620-5 (2009).

2. Evans, J. M., Donnelly, L. A., Emslie-Smith, A. M., Alessi, D. R. \& Morris, A. D. Metformin and reduced risk of cancer in diabetic patients. BMJ 330, 1304-5 (2005).

3. Dowling, R. J. et al. Changes in insulin receptor signaling underlie neoadjuvant metformin administration in breast cancer: a prospective window of opportunity neoadjuvant study. Breast Cancer Res. https://doi.org/10.1186/513058-015-0540-0 (2015).

4. Dowling, R. J., Niraula, S., Stambolic, V. \& Goodwin, P. J. Metformin in cancer: translational challenges. J. Mol. Endocrinol. 48, R31-43 (2012).

5. Pimentel, I. et al. A phase II randomized clinical trial of the effect of metformin versus placebo on progression-free survival in women with metastatic breast cancer receiving standard chemotherapy. Breast 48, 17-23 (2019).

6. Niraula, S. et al. Metformin in early breast cancer: a prospective window of opportunity neoadjuvant study. Breast Cancer Res. Treat. 135, 821-30 (2012).

7. Cazzaniga, M. et al. The effect of metformin on apoptosis in a breast cancer presurgical trial. Br. J. Cancer 109, 2792-7 (2013). 
8. Hadad, S. et al. Evidence for biological effects of metformin in operable breast cancer: a pre-operative, window-of-opportunity, randomized trial. Breast Cancer Res. Treatment. 128, 783-94 (2011).

9. Martin-Castillo, B. et al. A phase 2 trial of neoadjuvant metformin in combination with trastuzumab and chemotherapy in women with early HER-2 positive breast cancer: the METTEN study. Oncotarget 9, 35687-35704 (2018).

10. Goodwin, P. J. et al. Effect of metformin vs placebo on and metabolic factors in NCIC CTG MA.32. J. Natl Cancer Inst. https://doi.org/10.1093/JNCl/djv006 (2015).

11. Zhou, K. et al. Common variants near ATM are associated with glycemic response to metformin in type 2 diabetes. Nat. Genet. 43, 117-120 (2011).

12. Out, M. et al. A gene variant near ATM affects the response to metformin and metformin plasma levels; a post hoc analysis of an RCT. Pharmacogenomics 19, 715-726 (2018).

13. Florez, J. C. et al. The C allele of rs 11212617 does not associate with metformin response in the Diabetes Prevention Program. Diabetes Care 35, 1864-1867 (2012).

14. van Leeuwen, $\mathrm{N}$. et al. A gene variant near ATM is significantly associated with metformin treatment response in type 2 diabetes; a replication and meta-analysis of five cohorts. Diabetologia 55, 1971-1977 (2012).

15. Cuyas, E. et al. The C allele of ATM rs11212617 associates with higher pathological complete remission rate in breast cancer patients treated with neoadjuvant metformin. Front. Oncol. https://doi.org/10.3389/fonc.2019.00193 (2019).

16. Bonora, E. et al. Homeostasis model assessment closely mirrors the glucose clamp technique in the assessment of insulin sensitivity: studies in subjects with various degrees of glucose tolerance and insulin sensitivity. Diabetes Care. 23, 57-63 (2000).

17. Goodwin, P. J. et al. Insulin-lowering effects of metformin in women with early breast cancer. Clin. Breast Cancer 8, 501-5 (2008).

18. Kalinsky, K. et al. Presurgical trial of metformin in overweight and obese patients with newly diagnosed breast cancer. Cancer Investig. 32, 150-7 (2014).

19. DeCensi, A. et al. Differential effects of metformin on breast cancer proliferation according to markers of insulin resistance and tumor subtype in a randomized presurgical trial. Breast Cancer Res. Treat. 148, 81-90 (2014).

20. Williams, L. K. et al. Differing effects of metformin on glycemic control by raceethnicity. J. Clin. Endocrinol. Metab. 99, 3160-8 (2014).

21. Knowler, W. C. et al. Reduction in the incidence of type 2 diabetes with lifestyle intervention or metformin. N. Engl. J. Med. 346, 393-403 (2002).

22. Goodwin, P. J. et al. Metadata record for the article: Effect of Metformin versus Placebo on Metabolic Factors in the MA.32 Randomized Adjuvant Breast Cancer Trial. figshare https://doi.org/10.6084/m9.figshare.14447598 (2021).

\section{ACKNOWLEDGEMENTS}

This work was supported by the Canadian Cancer Society Research Institute (\#021039); National Cancer Institute (US) (\#CA077202, CA180868, CA180822); The Breast Cancer Research Foundation (New York); Canadian Breast Cancer Foundation Ontario Region, Ontario Institute for Cancer Research (\#10NOV-467); Canadian Institutes of Health Research (\#162296); Apotex Canada (in kind donation of placebo and metformin); and Hold'em for Life Charity. The study sponsors had no role in the design of the study; the collection, analysis, and interpretation of the data; the writing of the manuscript; or the decision to submit the manuscript for publication.

\section{AUTHOR CONTRIBUTIONS}

Study conception and design: P.J.G., R.J.O.D., B.E.C., W.R.P., L.E.S., K.A.G., J.A.L., D.L.H., I.A.M., T.J.W., T.J.H., P.R., M.R.-P., J.L., A.M.T., D.W.R. and V.S. Subject enrollment: P.J.G., W.R.P., M.J.B., R.V.M., A.M., A.G., K.A.G., J.A.L., D.L.H., I.A.M., T.J.H., T.J.W., P.R., M.R.-P., J.L., A.M.T. and D.W.R. Statistical analysis: B.E.C. and M.E. Writing and review of manuscript: All authors.

\section{COMPETING INTERESTS}

Dr. Whelan reports non-financial support from Genomic Health, outside the submitted work. Dr. Ryan Dowling is currently an employee at Hoffmann-La Roche Limited, Mississauga, ON. Dr. Julie Lemieux reports honoraria from Novartis, Pfizer, Eli Lilly. Dr. Ingrid Mayer reports honoraria from Novartis, grants and personal fees from Pfizer, grants and personal fees from Genentech, personal fees from Lilly, personal fees from Puma, personal fees from Abbvie, personal fees from Immunomedics, personal fees from Macrogenics, personal fees from Seattle Genetics, personal fees from Astra-Zeneca, personal fees from GSK. All of these are outside the submitted work. The other authors report no competing interests.

\section{ADDITIONAL INFORMATION}

Supplementary information The online version contains supplementary material available at https://doi.org/10.1038/s41523-021-00275-z.

Correspondence and requests for materials should be addressed to P.J.G.

Reprints and permission information is available at http://www.nature.com/ reprints

Publisher's note Springer Nature remains neutral with regard to jurisdictional claims in published maps and institutional affiliations.

(c) Open Access This article is licensed under a Creative Commons , which permits use, sharing, adaptation, distribution and reproduction in any medium or format, as long as you give appropriate credit to the original author(s) and the source, provide a link to the Creative Commons license, and indicate if changes were made. The images or other third party material in this article are included in the article's Creative Commons license, unless indicated otherwise in a credit line to the material. If material is not included in the article's Creative Commons license and your intended use is not permitted by statutory regulation or exceeds the permitted use, you will need to obtain permission directly from the copyright holder. To view a copy of this license, visit http://creativecommons. org/licenses/by/4.0/.

(c) The Author(s) 2021 